\title{
Prasugrel-Induced Hypersensitivity Skin Reaction
}

\author{
Soo-Han Kim, MD, Sang-Don Park, MD, Yong-Soo Baek, MD, Seon-Young Lee, MD, \\ Sung-Hee Shin, MD, Sung-II Woo, MD, Dae-Hyeok Kim, MD, and Jun Kwan, MD \\ Division of Cardiology, Department of Internal Medicine, Inha University College of Medicine, Incheon, Korea
}

We report a case of hypersensitivity skin reaction to prasugrel. The patient exhibited a generalized skin rash after treatment with prasugrel, which was resolved after discontinuation of prasugrel and substitution to clopidogrel. Clopidogrel was successfully administered as an alternative to prasugrel without any signs of further hypersensitivity. (Korean Circ J 2014;44(5):355-357)

KEY WORDS: Prasugrel; Clopidogrel; Thienopyridine; Hypersensitivity; Exanthema.

\section{Introduction}

Prasugrel is an anti-platelet thienopyridine agent." In vivo, the metabolites of prasugrel irreversibly bind to the P2Y class of adenosine diphosphate receptors on platelets and inhibit platelet activation and aggregation. Prasugrel was approved by the Food and Drug Administration in July 2009 after it was shown to benefit patients undergoing percutaneous coronary intervention (PCI) by reducing thrombotic complications. ${ }^{2)}$ Adverse hypersensitivity reactions to clopidogrel are well described and occur in about 1\% of patients, of which 93\% present with a maculopapular rash that commonly leads to discontinuation of the drug. ${ }^{3 / 4)}$ Prasugrel is structurally similar to clopidogrel, and thus it is reasonable to surmise that there may be cross-reactivity between these two compounds. However, there is currently little data with respect to the existence of such hypersensitivity skin reactions to prasugrel alone or cross-reactivity to clopidogrel. ${ }^{2)}$ Here, we report the case of a patient with a prasugrel induced skin rash that was resolved after substitution of prasugrel to clopidogrel.

Received: August 21, 2013

Revision Received: September 4, 2013

Accepted: October 8, 2013

Correspondence: Soo-Han Kim, MD, Division of Cardiology, Department of Internal Medicine, Inha University Hospital, 27 Inhang-ro, Jung-gu, Incheon 400-711, Korea

Tel: 82-32-890-2441, Fax: 82-32-890-2447

E-mail: naeaidi@hanmail.net

- The authors have no financial conflicts of interest.

This is an Open Access article distributed under the terms of the Creative Commons Attribution Non-Commercial License (http://creativecommons. org/licenses/by-nc/3.0) which permits unrestricted non-commercial use, distribution, and reproduction in any medium, provided the original work is properly cited.

\section{Case}

A 41-year-old male visited the EMC of our hospital complaining of a sharpening chest pain of his mid sternum lasting for 1 hour. He had previously been diagnosed with ankylosing spondylitis and had been treated with oral meloxicam, sulfasalazine, and methylprednisolone for 10 years. The patient had also been diagnosed with hypertension for 8 years, but did not take any anti-hypertensive medications. EKG revealed ST-segment elevations in the $\mathrm{V}$ 2-4 precordial leads. A chest $X$-ray revealed mild pulmonary congestion and laboratory tests revealed elevated leukocytes, cardiac enzymes, and Nterminal b-natriuretic peptide levels. The patient was subsequently diagnosed with ST elevation myocardial infarction, which we addressed by performing an emergent $\mathrm{PCl}$. Before a coronary angiography was performed, we administrated a loading dose of clopidogrel 600 mg, aspirin 300 mg, and carvedilol. Coronary angiography revealed subtotal occlusion in the proximal left anterior descending artery (LAD). PCl using drug-eluting stents (DESs; Xience prime, Abbott Vascular, Santa Clara, CA, USA, 3.0×38 in proximal to mid LAD lesion, $2.5 \times 15$ in the 1st diagonal branch lesion) was performed successfully without any significant complications. A rheumatologist confirmed that the patient was not in the course of active disease, and thus we decided to withhold rheumatologic medications in order to focus on treating the myocardial infarction. The treatment plan for the patient consisted of continued oral methylprednisolone to control ankylosing spondylitis, and we switched clopidogrel to prasugrel, which is known as a more potent thrombus inhibitor, on the 3rd day of admission considering the increased risk of stent thrombosis. Prasugrel was administered with a loading dose of $50 \mathrm{mg}$ daily maintenance dose of $10 \mathrm{mg}$. The patient was discharged taking aspirin, carvedilol, rosuvastatin, candesartan, 

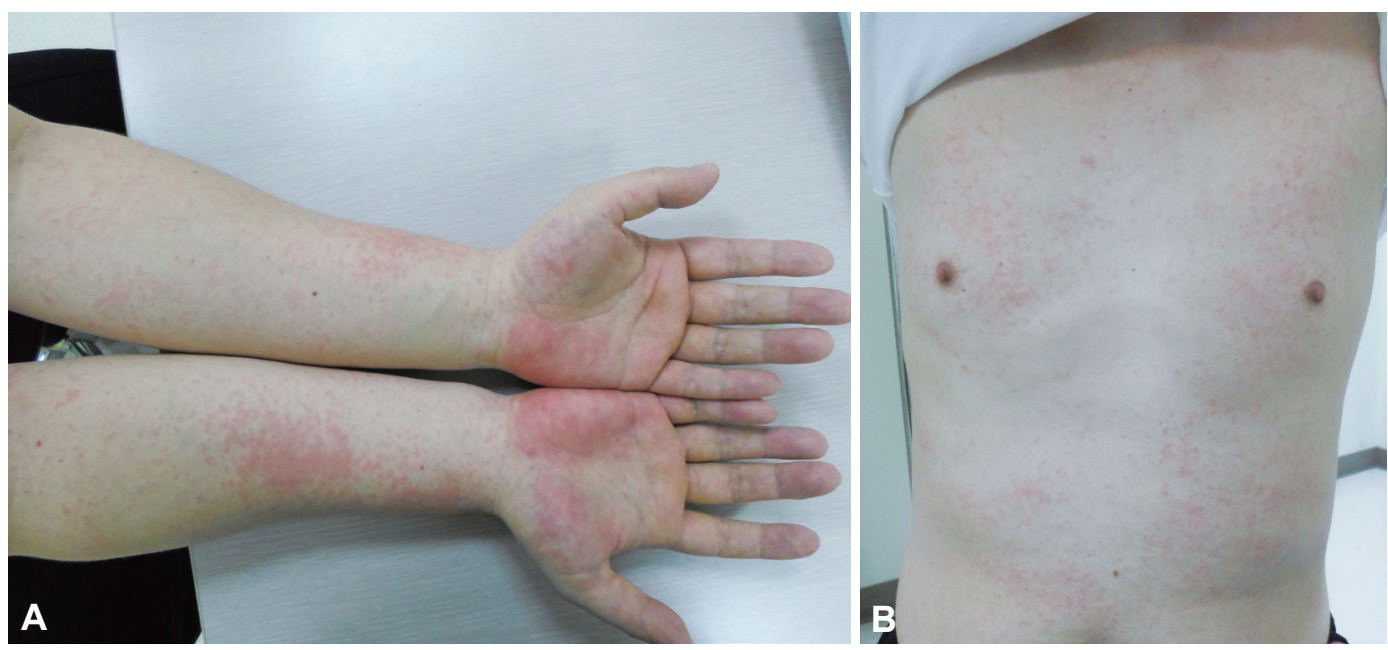

Fig. 1. Photographs of patient with prasugrel-induced hypersensitivity skin reaction. A: exanthema on both forearms and hands. (A) shows exanthema on both forearms and hands after 5 days of prasugrel exposure. B: exanthema on the anterior chest and abdominal wall. (B) shows exanthema on anterior chest and abdominal wall.

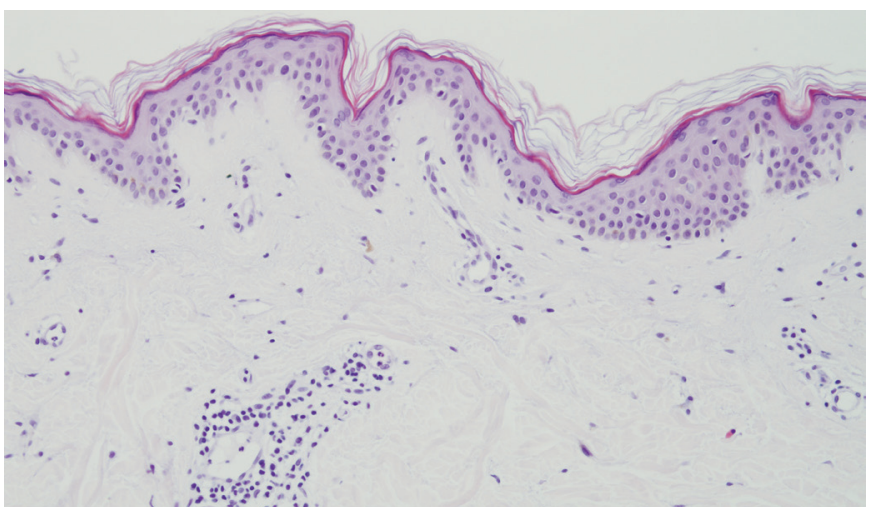

Fig. 2. Microscopic section of punch biopsy of abdominal wall. Microscopic sections demonstrating perivascular lymphocyte infiltration of dermis and epidermis compatible with a hypersensitivity skin reaction.

furosemide, spironolactone and prasugrel; however, he revisited our outpatient clinic after 5 days complaining of a worsened whole body skin rash with pruritic symptoms (Fig. 1). Blood tests and chest $\mathrm{X}$-rays showed no abnormal findings, and vital signs were within normal limits. Because aspirin was administered without any evidence of skin hypersensitivity or reaction prior to admission, we suspected prasugrel as the cause of the skin reaction. Accordingly, we discontinued prasugrel and switched the patient to clopidogrel $(75 \mathrm{mg} / \mathrm{d})$. A punch biopsy of the abdominal skin revealed mild perivascular lymphocytic infiltrations that were compatible with a hypersensitivity skin reaction (Fig. 2). The patient was followed through the outpatient clinic without further complaint of hypersensitivity skin reaction.

\section{Discussion}

Dual antiplatelet therapy with aspirin and thienopyridine plays a pivotal role in preventing stent thrombosis among patients undergoing PCls. ${ }^{5)}$ Prasugrel is a 3rd generation thienopyridine that was recently shown to be superior to clopidogrel for preventing stent thrombosis in the Assess Improvement in Therapeutic Outcomes by Optimizing Platelet Inhibition with Prasugrel-Thrombolysis in Myocardial Infarction 38 (TRITON-TIMI 38) trial. ${ }^{1)}$ According to the TRITON-TIMI 38 trial, the incidence of skin rashes is slightly higher in patients treated with prasugrel (2.8\%) than with clopidogrel (2.4\%)." When a hypersensitivity skin reaction with clopidogrel is observed, several treatment options are available including desensitization to clopidogrel, substitution to another thienopyridine, and anticoagulation. ${ }^{3)}$ However, guidelines for managing prasugrel induced hypersensitivity skin reaction in patients with DES have not yet been established.

To date there have been two case reports of prasugrel induced skin rashes. Raccah et al. ${ }^{6}$ was the first to report a prasugrel induced skin rash. ${ }^{3)}$ The patient they described had a prior history of hypersensitivity to clopidogrel, and thus was switched from prasugrel to clopidogrel combined with a glucocorticoid and an anti-histamine. Abhishek et al. ${ }^{2)}$ also reported subsidence of a prasugrel induced skin rash after substitution with clopidogrel. ${ }^{2)}$ Based on the treatment options for clopidogrel hypersensitivity and previous reports, several alternative treatment options can be considered in cases of prasugrel induced hypersensitivity reaction. The first option is to use ticlopidine. ${ }^{3)}$ Unfortunately, ticlopidine is associated with several common hematologic adverse events, including neutropenia, and is less effective than thienopyridine for preventing atherothrombotic events and stent thrombosis. The second option is anticoagulation with warfarin,;) however, warfarin is less effective than thienopyridines for reducing stent thrombosis, ${ }^{6}$ and significantly increases the risk of major bleeding. The third option is desensitization to prasugrel. 
Mutnick ${ }^{7)}$ reported the first case of successful desensitization to prasugrel using a 7-hour desensitization protocol. However, because the prasugrel desensitization protocol includes a drug washout period, this method cannot be used routinely after DES stent implantation because of the increased risk of acute stent thrombosis. In addition, desensitization is time-consuming and labor-intensive, and thus this method is not widely used in clinical practice. A final option is to use another anti-platelet agent such as ticagrelor and clopidogrel. Ticagrelor is a new non-thienopyridine P2Y12 inhibitor ${ }^{3)}$ belonging to the cyclopentyl-triazolo-pyrimidine class of drugs, the structure and $\mathrm{P} 2 \mathrm{Y} 12$ receptor binding site of which are distinct from thienopyridine. As a result, ticagrelor is a good alternative for addressing thienopyridine induced skin rashes. In our case, we were able to resolve the skin hypersensitivity reaction by switching from prasugrel to clopidogrel. The ability of clopidogrel to prevent stent thrombosis has been demonstrated in numerous clinical studies, ${ }^{1) 5}$ and thus clopidogrel may be the best treatment option for prasugrel induced hypersensitivity skin reactions. However, prasugrel and clopidogrel have similar structures and mechanisms of action, and thus one can surmise the possibility of cross-reactivity between these two drugs. Unfortunately, there is little data concerning the possible cross-reactivity between these two drugs. ${ }^{48)}$ Accordingly, physicians should keep in mind the possible cross-reactivity between clopidogrel and prasugrel when switching between these drugs.

\section{References}

1. Wiviott SD, Braunwald E, McCabe $C$, et al. Prasugrel versus clopidogrel in patients with acute coronary syndromes. N Engl J Med 2007;357: 2001-15.

2. Deshmukh AJ, Pant $S$, Cook J, Sachdeva R, Rutlen D, Uretsky BF. Prasugrel-induced rash. Ann Pharmacother 2012;46:1123-5.

3. Lokhandwala J, Best PJ, Henry Y, Berger PB. Allergic reactions to clopidogrel and cross-reactivity to other agents. Curr Allergy Asthma Rep 2011;11:52-7.

4. Lokhandwala JO, Best PJ, Butterfield JH, et al. Frequency of allergic or hematologic adverse reactions to ticlopidine among patients with allergic or hematologic adverse reactions to clopidogrel. Circ Cardiovasc Interv 2009;2:348-51.

5. Mehta SR, Yusuf S, Peters RJ, et al. Effects of pretreatment with clopidogrel and aspirin followed by long-term therapy in patients undergoing percutaneous coronary intervention: the PCI-CURE study. Lancet 2001;358:527-33.

6. Bertrand ME, Legrand V, Boland J, et al. Randomized multicenter comparison of conventional anticoagulation versus antiplatelet therapy in unplanned and elective coronary stenting. The full anticoagulation versus aspirin and ticlopidine (fantastic) study. Circulation 1998;98: 1597-603.

7. Mutnick JL. Desensitization to prasugrel: cardiology's increased need for allergy consultation. Ann Allergy Asthma Immunol 2012;108:124-5.

8. Cheema AN, Mohammad A, Hong $T$, et al. Characterization of clopidogrel hypersensitivity reactions and management with oral steroids without clopidogrel discontinuation. J Am Coll Cardio/ 2011;58:1445-54. 